\title{
THE EFFECT OF RELATIVE HUMIDITY ON THE FLASHOVER STRENGTH OF SOLID INSULATION
}

\author{
R. W. Macpherson $\xi$, M. P. Wilson, I. V. Timoshkin, S. J. MacGregor, M. J. Given \\ Dept. Electronic \& Electrical Engineering, University of Strathclyde, 204 George Street, \\ Glasgow, G1 1XW, UK
}

\begin{abstract}
This paper informs on the flashover strength of 3 materials: Delrin (Polyoxymethylene), HDPE (High Density Polyethylene) and Ultem (Polyetherimide) with 'smooth' and 'knurled' surface finishes, in zero-grade air at $-0.5,0$ and 0.5 bar gauge, and at $<10 \%, \sim 50 \%$ and $>90 \%$ relative humidity $(\mathrm{RH})$. All tests were completed using a 10-stage Marx generator, producing HV impulses with a nominal $100 / 700 \mathrm{~ns}$ waveshape. Each test conformed with the ASTM D3426-97 standard of 'step up' testing, to find the average flashover voltage for each set of conditions. The electrode system with each dielectric material demonstrated a decrease in breakdown voltage as the $\mathrm{RH}$ was increased. In high humidity environments, the knurled surface finish was seen to have a positive effect on the flashover strength of Delrin and Ultem samples, compared to a smooth, machined surface. Increasing pressure yielded an increase in the flashover strength also. For samples with a smooth surface finish, a decrease in flashover strength was found as the permittivity of the material increased, irrespective of humidity and pressure. However, a knurled surface finish had a much more erratic effect on flashover strength, with no discernible trend with increasing material permittivity.
\end{abstract}

\section{I.INTRODUCTION}

Within pulsed power systems, the governing factor determining the overall breakdown strength of the system is often the voltage that initiates flashover, in the vicinity of solid insulating parts required to provide mechanical support. When solid insulation is surrounded by air, changes in the air humidity are known to have an effect on the flashover strength of different composite insulation systems. Experimental results are reported in [1], for example, with surface flashover events characterised in air with varying humidity for PTFE, Silicon Rubber (SIR), Nylon and Glazer Porcelain, with a rod-plane electrode topology, under positive impulse voltages. The authors observed that, as the absolute humidity increased from 5 to $25 \mathrm{~g} / \mathrm{m}^{3}$, the flashover voltage was seen to increase for both lightning impulse (LI) and switching impulse (SI) voltages.

\footnotetext{
६ email: ruairidh.macpherson@strath.ac.uk
}

In [2], surface flashover of solids was investigated, with the effect of varying air humidity and surface roughness of the insulators characterised. The authors reported that, as humidity was increased, there were no changes in either the $\mathrm{AC}$ or impulse flashover strength of the composite insulation system, for humidity levels ranging from 474 to $1438 \mathrm{ppm}$. However, as surface roughness was increased from 1.6 to $25 \mu \mathrm{m}$, the flashover strength of the insulation was negatively affected, decreasing by $\sim 7 \%-22 \%$ under negative polarity.

In [3], flashover of air gaps without solid spacers was characterised, under high humidity levels, up to and including $100 \%$ relative humidity. Under these conditions, fog accumulation was found to have a detrimental effect on the breakdown strength of the air gap tested, especially for values of $\mathrm{RH}>80 \%$.

In this work, experimental data has been generated on the flashover strength of three solid materials, Delrin (Polyoxymethylene), HDPE (High Density Polyethylene) and Ultem (Polyetherimide). Samples of these materials were machined into the form of rods $40 \mathrm{~mm}$ in length and $30 \mathrm{~mm}$ in diameter, with either a 'smooth' machined surface finish, or a modified 'knurled' finish, consisting of angled diamond indentations over the surface of the material. The tests were conducted at values of $<10 \%$, $\sim 50 \%$ and $>90 \%$ relative humidity $(\mathrm{RH})$, and at pressures of $-0.5,0$ and 0.5 bar gauge. Samples were subjected to negative polarity impulsive voltages from a 10-stage Marx generator, with 100/700 ns wave shape. The relative permittivity of each of the materials is $\varepsilon_{r}=2.3$ for HDPE [4], $\varepsilon_{r}=3.0$ for Ultem [5], and $\varepsilon_{r}=3.8$ for Delrin [6]. The $U_{50}$ flashover initiation voltages from the resulting waveforms were determined and the standard deviation was calculated from each data set of 20 breakdown events. Thus, the flashover strength of samples of the three different materials could be compared at different levels of RH, different pressures, and with different surface finishes.

\section{II.EXPERIMENTAL ARRANGEMENT}

In Figure 1, the test cell used has been illustrated, with the dimensions of each component part. The electrodes shown, made of stainless-steel and polished to a mirror finish, resulted in a quasi-uniform electrical field 
distribution. The gas to be tested was input via an inlet at the bottom of the test cell. A humidity sensor was added in order to allow for the RH of the gas to be monitored.

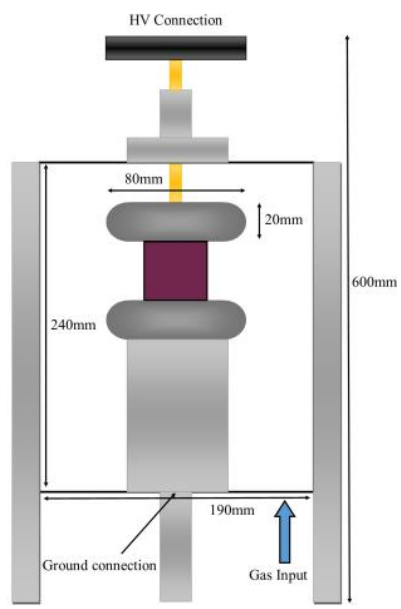

Figure 1. Schematic diagram of test cell, showing spacer insertion, dimensions and connections.

The charging voltage was supplied from a $100 \mathrm{kV}, 2.5$ $\mathrm{mA}$, Glassman high voltage DC power supply, connected through a $1 \mathrm{M} \Omega$ charging resistor. This was then connected to the output of the 10-stage Marx generator. The $\mathrm{CuSO}_{4}$ wave-shaping resistors had values of $300 \Omega$ for the wave tail and $700 \Omega$ for the output resistor. This specific resistor arrangement resulted in a 100/700 ns output voltage waveform. The output of the Marx generator was connected to the test cell and a voltage divider in parallel. A 1000:1 Tektronix P6015A HV probe was connected to the output of an $8: 1 \mathrm{CuSO}_{4}$ resistive voltage divider, and through to a Tektronix MDO3012 oscilloscope for analysis of the resulting waveforms.

Several stages were incorporated into the gas-handling system, allowing the relative humidity of the gas in the test cell to be altered and monitored prior to testing. This was achieved using a 'wet chamber', connected to a gas distribution board, where air can be passed through a chamber filled with distilled water and an ultrasonic humidifier. The output of the gas bottle is connected to both, a 'dry line' and a 'wet line', allowing for $<10 \%$, $\sim 50 \%$ and $>90 \% \mathrm{RH}$ to be achieved. The achieved humidity was then monitored by a TE Connectivity HPP801A031 humidity sensor, the output capacitance of which changes with RH, housed within the test cell. This was connected via a buffer circuit, with changes in the frequency of the output signal corresponding to changes in RH, to a separate Rohde and Schwarz HMO2024 oscilloscope, allowing the humidity to be monitored throughout the testing process.

The testing procedure implemented was a 'step up' method, as included in the ASTM D3426-97 standard [7]. The voltage level initially applied was set to provide a low probability of flashover, before the charging voltage was increased in iterations of $300 \mathrm{~V}$, monitored on a DMM though a 1000:1 Testec HVP-40 HV probe. Once a flashover event was initiated, the resulting waveform was inspected, and the flashover voltage recorded. The output voltage was then decreased to a level with a low probability of breakdown, and the process repeated until the occurrence of another flashover event. Two, clear withstand levels were always observed before a valid breakdown voltage was recorded. This was conducted 20 times for each set of test conditions, in order to find the $U_{50}$ flashover initiation voltage for each of the tests.

\section{III.EXPERIMENTAL RESULTS}

The generated experimental data is separated by $\mathrm{RH}$ level, enabling comparison of the performance of the three materials at different pressures, and with different surface finishes. Figures 2, 3 and 4 show the graphical breakdown voltage data for $<10 \% \mathrm{RH}, \sim 50 \% \mathrm{RH}$ and $>90 \% \mathrm{RH}$, respectively, showing the average flashover voltages and standard deviations (error bars). Tables 1, 2 and 3 show the numerical information for each material at $-0.5,0$, and 0.5 bar gauge, respectively, for ease of comparison. Each breakdown voltage value is an average of 20 breakdown events. Error bars in the figures and \pm values in the tables show standard deviation.

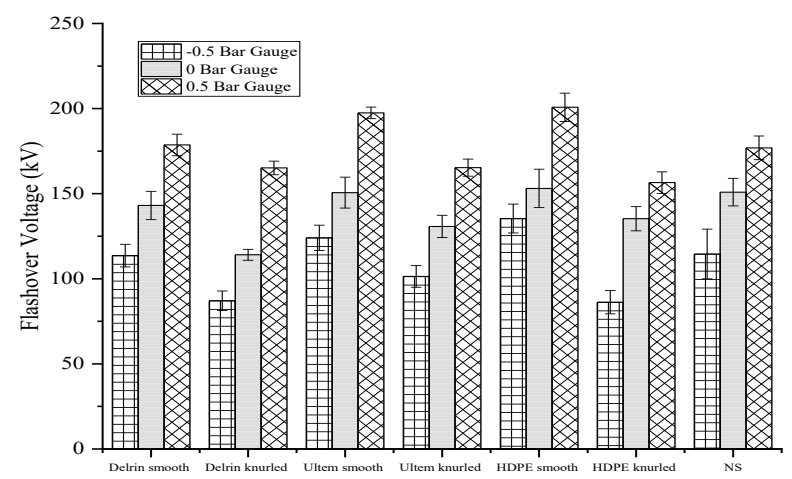

Figure 2. Average flashover voltages for Delrin, Ultem and HDPE samples with both smooth and knurled finishes, and at pressures of $-0.5,0$ and 0.5 bar gauge, for $<10 \%$ RH; NS: no spacer test.

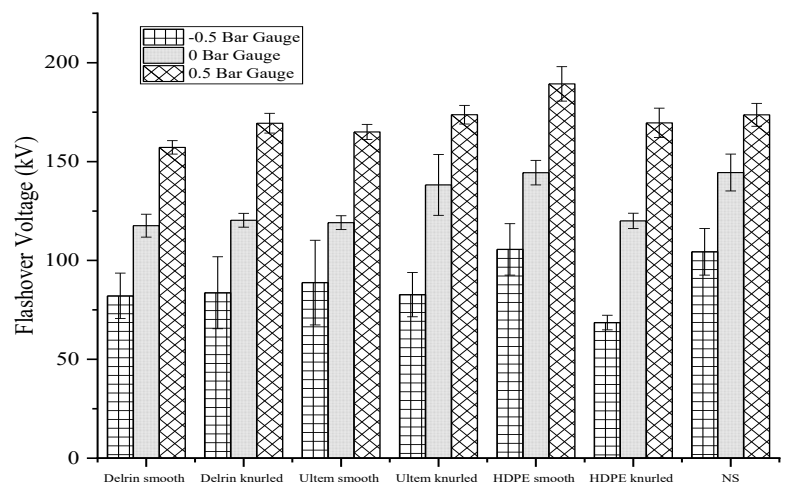

Figure 3. Average flashover voltages for Delrin, Ultem and HDPE samples with both smooth and knurled finishes, and at pressures of $-0.5,0$ and 0.5 bar gauge, for $\sim 50 \%$ RH; NS: no spacer test. 


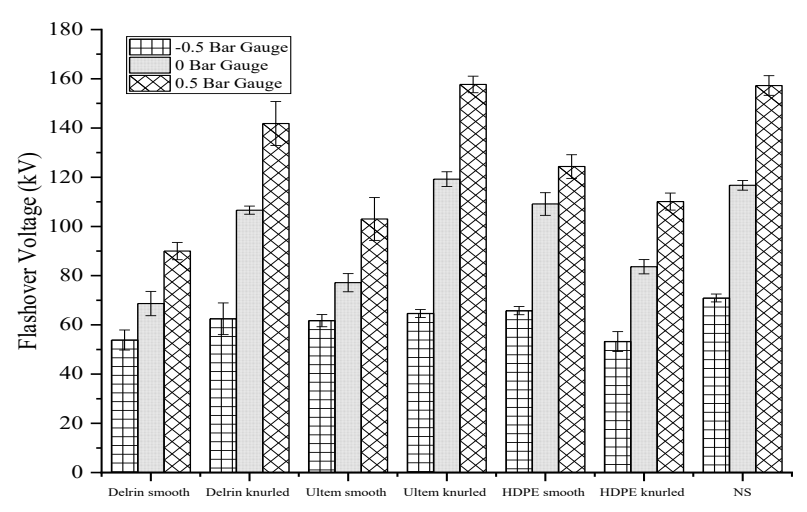

Figure 4. Average flashover voltages for Delrin, Ultem and HDPE samples with both smooth and knurled finishes, and at pressures of $-0.5,0$ and 0.5 bar gauge, for $>90 \%$ RH; NS: no spacer test.

Table 1. $U_{50}$ flashover voltages at -0.5 bar gauge for samples of the three tested materials, with varying surface finish and $\mathrm{RH}$.

\begin{tabular}{|l|l|l|l|}
\hline & $\begin{array}{c}\mathbf{U}_{\mathbf{5 0}}<\mathbf{1 0 \%} \\
\mathbf{R H}(\mathbf{k V})\end{array}$ & $\begin{array}{c}\mathbf{U}_{\mathbf{5 0}} \sim \mathbf{5 0} \% \\
\mathbf{R H} \mathbf{( k V )}\end{array}$ & $\begin{array}{c}\mathbf{U}_{\mathbf{5 0}} \boldsymbol{> 9 0 \%} \\
\mathbf{R H}(\mathbf{k V})\end{array}$ \\
\hline No Spacer & $114.6 \pm 14.6$ & $104.4 \pm 11.8$ & $70.9 \pm 1.6$ \\
\hline Delrin Smooth & $113.6 \pm 6.7$ & $82.1 \pm 11.5$ & $53.9 \pm 4.1$ \\
\hline Delrin Knurled & $87.1 \pm 5.8$ & $83.7 \pm 18.2$ & $62.5 \pm 6.4$ \\
\hline Ultem Smooth & $124.1 \pm 7.4$ & $88.8 \pm 21.4$ & $61.7 \pm 2.5$ \\
\hline Ultem Knurled & $101.4 \pm 6.4$ & $82.7 \pm 11.2$ & $64.6 \pm 1.6$ \\
\hline HDPE Smooth & $135.4 \pm 8.5$ & $105.6 \pm 13.0$ & $65.8 \pm 1.7$ \\
\hline HDPE Knurled & $86.3 \pm 6.9$ & $68.6 \pm 3.7$ & $53.2 \pm 4.1$ \\
\hline
\end{tabular}

Table 2. $U_{50}$ flashover voltages at 0 bar gauge for samples of the three tested materials, with varying surface finish and RH.

\begin{tabular}{|l|l|l|l|}
\hline & \multicolumn{1}{|c|}{$\begin{array}{c}\mathbf{U}_{\mathbf{5 0}}<\mathbf{1 0 \%} \\
\mathbf{R H}(\mathbf{k V})\end{array}$} & $\begin{array}{c}\mathbf{U}_{\mathbf{5 0}} \sim \mathbf{5 0 \%} \\
\mathbf{R H}(\mathbf{k V})\end{array}$ & \multicolumn{1}{|c|}{$\begin{array}{c}\mathbf{U}_{\mathbf{5 0}}>\mathbf{9 0} \% \\
\mathbf{R H}(\mathbf{k V})\end{array}$} \\
\hline No Spacer & $150.9 \pm 8.1$ & $144.5 \pm 9.3$ & $116.7 \pm 2$ \\
\hline Delrin Smooth & $143.1 \pm 8.3$ & $117.6 \pm 5.8$ & $68.7 \pm 4.9$ \\
\hline Delrin Knurled & $114.1 \pm 3.2$ & $120.3 \pm 3.5$ & $106.1 \pm 6.4$ \\
\hline Ultem Smooth & $150.6 \pm 9.1$ & $119.1 \pm 3.5$ & $77.2 \pm 3.7$ \\
\hline Ultem Knurled & $130.8 \pm 6.5$ & $138.2 \pm 15.4$ & $119.2 \pm 3$ \\
\hline HDPE Smooth & $153.1 \pm 11.3$ & $144.5 \pm 6.2$ & $109.1 \pm 4.6$ \\
\hline HDPE Knurled & $135.3 \pm 7.1$ & $120 \pm 3.9$ & $83.6 \pm 2.9$ \\
\hline
\end{tabular}

Table 3. $U_{50}$ flashover voltages at 0.5 bar gauge for samples of the three tested materials, with varying surface finish and $\mathrm{RH}$.

\begin{tabular}{|l|c|c|c|}
\hline & $\begin{array}{c}\mathbf{U}_{\mathbf{5 0}}<\mathbf{1 0 \%} \\
\mathbf{R H}(\mathbf{k V})\end{array}$ & $\begin{array}{c}\mathbf{U}_{\mathbf{5 0}} \sim \mathbf{5 0 \%} \\
\mathbf{R H}(\mathbf{k V})\end{array}$ & $\begin{array}{c}\mathbf{U}_{\mathbf{5 0}} \boldsymbol{> 9 0 \%} \\
\mathbf{R H}(\mathbf{k V})\end{array}$ \\
\hline No Spacer & $176.9 \pm 6.9$ & $173.7 \pm 5.8$ & $157.3 \pm 4$ \\
\hline Delrin Smooth & $178.6 \pm 6.3$ & $157.2 \pm 3.4$ & $90 \pm 3.5$ \\
\hline Delrin Knurled & $165.1 \pm 4.0$ & $169.5 \pm 5.0$ & $141.8 \pm 8.9$ \\
\hline Ultem Smooth & $197.5 \pm 6.5$ & $165.0 \pm 3.8$ & $103.1 \pm 8.7$ \\
\hline Ultem Knurled & $165.3 \pm 4.9$ & $173.7 \pm 4.7$ & $157.7 \pm 3.4$ \\
\hline HDPE Smooth & $200.8 \pm 8.3$ & $189.6 \pm 8.7$ & $124.3 \pm 4.8$ \\
\hline HDPE Knurled & $156.5 \pm 6.3$ & $169.6 \pm 7.4$ & $110.1 \pm 3.5$ \\
\hline
\end{tabular}

Figures (2-4) show the average flashover voltages and standard deviations calculated from the gathered data. Firstly, in all tests conducted, as the pressure was increased the flashover voltage increased also. As the gas pressure increases, the electron mean free path decreases, and the collision frequency increases. Electrons will gain less energy between collisions, which means that a higher applied field is required to allow the electrons to gain sufficient energy in order to cause an ionization event [8].

For all sets of experimental conditions, the breakdown strength of the insulation system decreased with increasing humidity. As the humidity increases, water accumulates on the spacer surfaces, creating a more conductive surface over the length of the solid dielectric [9]. Therefore, the surface resistivity of the insulating spacer will decrease, and the flashover voltage will decrease accordingly, as seen from the test results in Figures (2-4) and Tables (1-3).

The maximum average flashover voltage of $\sim 200 \mathrm{kV}$ achieved in the tests was with an HDPE spacer with smooth surface finish, at 0.5 bar gauge and $<10 \% \mathrm{RH}$. The minimum average flashover voltage of $\sim 53 \mathrm{kV}$ was recorded for two different solid samples, both at -0.5 bar gauge and $>90 \% \mathrm{RH}$ : for an HDPE spacer with a knurled surface finish; and for a Delrin spacer with a smooth surface finish.

Across all tests, an increase in RH had an adverse effect on the flashover strength of the insulation system. Firstly, the effect of the RH can be seen in the no spacer ('NS') tests. A decrease in average breakdown voltage was seen as the relative humidity was increased, especially from $\sim 50 \%$ to $>90 \%$, as the distruptive-discharge voltage becomes irregular [10]. At $>90 \% \mathrm{RH}$, the test cell was encapsulated in a fog-like environment, resulting in a significant drop in the breakdown strength of the air gap, seen also in [3] and [11].

When solid spacers were included between the electrodes, the reductions in the average flashover voltage with increasing relative humidity were greater for smooth surface finishes than for knurled surface finishes. At 0.5 bar gauge (Table 3), for smooth surface finishes, the average flashover voltages fell by $\sim 77 \mathrm{kV}$ (38\%) for HDPE, by $\sim 89 \mathrm{kV}(50 \%)$ for Delrin, and by $\sim 94 \mathrm{kV}$ $(48 \%)$ for Ultem, respectively, when the humidity level was increased from $<10 \% \mathrm{RH}$ to $>90 \% \mathrm{RH}$. For knurled surface finishes, the corresponding decreases were $\sim 46 \mathrm{kV}(30 \%)$ for HDPE, $\sim 23 \mathrm{kV}$ (14\%) for Delrin, and $\sim 8 \mathrm{kV}(5 \%)$ for Ultem.

In terms of surface finish, the behaviour of the average flashover voltage varied at different levels of relative humidity, and for the different materials. At $<10 \% \mathrm{RH}$ and 0.5 bar gauge (Table 3), a knurled surface finish resulted in decreases in the average flashover voltage of $\sim 44 \mathrm{kV}(22 \%)$ for HDPE, $\sim 14 \mathrm{kV}(8 \%)$ for Delrin, and $\sim 32 \mathrm{kV}(16 \%)$ for Ultem, compared to samples of the same materials with smooth surface finishes. At $\sim 50 \%$ and $>90 \%$ RH, however, some spacers with knurled surface finishes were found to have increased average flashover voltages compared to those of the same material type with smooth surface finishes, by over $50 \mathrm{kV}$ for both Ultem and Delrin at 0.5 bar gauge (Table 3 ). However, for HDPE, the average flashover voltage with smooth surface finish was always greater than for knurled surface finish. 
The increase in flashover strength of Ultem and Delrin samples with knurled finishes in a high humidity environment is hypothesised as being due to an increased path length associated with breakdown, due to the values of absorbance (\% weight gain at saturation in water at $23{ }^{\circ} \mathrm{C}$ ) of the materials, quoted in [5] and [6], respectively. HDPE has a low value of absorbance [4], leading to a hydrophobic effect on surface water accumulation, enhancing the electrical field intensity over the surface of the material due to high local field regions and leading to breakdown. For Delrin and Ultem samples with knurled finishes at 0 bar gauge; and for Delrin, Ultem and HDPE samples with knurled finishes at 0.5 bar gauge; a slight increase in average breakdown voltage at $\sim 50 \% \mathrm{RH}$ was seen, compared to that at $<10 \% \mathrm{RH}$. Under medium humidity $(\sim 50 \% \mathrm{RH})$, the spark path is closer to the spacer itelf, whereas under low humidity $(<10 \% \mathrm{RH})$, the breakdown channel develops through the bulk of the air and the spark path is generally observed further away from the spacer.

At high ( $>90 \% \mathrm{RH})$ levels of humidity, higher average flashover voltages were rercorded for Delrin and Ultem samples with knurled surface finishes, than for those with smooth (machined) surface finishes. As the surface conductivity of the spacers will be increased by the surface water accumulation, one possible explanation for this behaviour is that there is a higher probability of a flashover across the solid surface/air interface than for bulk breakdown of the gas. It is hypothesised that this gives the advantages of the longer path length due to the knurled finish, resulting in higher flashover voltages than for samples with a smooth surface finish.

\section{IV.CONCLUSIONS AND FURTHER WORK}

From the results presented herein, it can be seen that increased relative humidity has an adverse effect on the average breakdown voltage for most sets of conditions tested. The highest recorded average flashover voltage of $\sim 200 \mathrm{kV}$ across all of the tests was for an HDPE spacer with a smooth surface finish, at 0.5 bar gauge and $<10 \%$ RH. The surface finish of the spacer was seen to have the opposite effect on flashover strength at $\sim 50 \%$ and $>90 \%$ RH for Ultem and Delrin compared to that at $<10 \%$ RH. Whether or not a knurled surface finish can enhance the performance of the overall insulation system is, therefore, dependent upon the expected environmental conditions during operation of the system.

Further work will consist of performing 3-parameter Weibull statistical analysis for each of the tests conducted, to understand the probability of breakdown associated with each set of test conditions. Also, as an increase in average breakdown voltage was seen to occur for some samples with knurled surface finishes compared to those with smooth, machined, surface finishes at high levels of humidity, more tests will be completed under this $(>90 \%$ $\mathrm{RH})$ regime, in order to further understand the mechanism of breakdown.

\section{V.REFERENCES}

[1] L. A. Lazaridis and P. N. Mikropoulos, "Positive impulse flashover along smooth cylindrical insulating surfaces under variable humidity," in IEEE Transactions on Dielectrics and Electrical Insulation, vol. 18, no. 3, pp. 745-754, June 2011.

[2] J. Seong et al., "Effect of humidity and electrode roughness on the $\mathrm{AC}$ and impulse breakdown characteristics of dry-air," 2012 IEEE International Conference on Condition Monitoring and Diagnosis, Bali, 2012, pp. 770-773.

[3] H. Niu and T. Xu, "The relevance between dramatic declines of air gap breakdown voltage and fog-haze weather," TENCON 2015 - 2015 IEEE Region 10 Conference, Macao, 2015, pp. 1-4.

[4] http://plastimnew.websitedesigntest.co.uk/content/upl oads/library/technical_datasheets/HDPE-PE\%20300-

Technical-Data-Sheet.pdf HDPE Data Sheet Accessed $11 / 6 / 2019$.

[5] https://www.plasticstockist.com/downloads/datasheet s/pei1000.pdf Ultem Data Sheet Accessed 11/6/2019.

[6] https://www.theplasticshop.co.uk/plastic_technical_d ata_sheets/delrin_acetal_homopolymer_technical_data_sh eet.pdf Delrin Data Sheet Accessed 11/6/2019.

[7] ASTM D3426-97 Standard Test Method for Dielectric Breakdown Voltage and Dielectric Strength of Solid Electrical Insulating Materials Using Impulse Waves.

[8] L. G. Christophorou and L. A. Pinnaduwage, "Basic physics of gaseous dielectrics" in IEEE Transactions on Electrical Insulation, vol. 25, no. 1, pp. 55-74, Feb 1990.

[9] R. K. Raiput “A textbook pf electrical engineering materials", Page 171, Firewall Media, 2004.

[10] IEC 60060-1; High-voltage test techniques - Part 1: General definitions and test requirements.

[11] N. Haiqing, G. Ran, X. Tao and X. Jia, "Research on weather condition of significant decline of air gap breakdown voltage," 2015 IEEE 15th International Conference on Environment and Electrical Engineering (EEEIC), Rome, 2015, pp. 1725-1729. 